\title{
Isolation and characterization of microsatellite loci in the Malaysian giant freshwater prawn, Macrobrachium rosenbergii
}

\begin{abstract}
Eight single locus microsatellite markers were developed to characterize the Malaysian giant freshwater prawn, Macrobrachium rosenbergii. These microsatellites were isolated from an enriched genomic library contained by using a 5 Alinchored polymerase chain reaction technique. Primers were designed to flank the repeat sequences and subsequently used to characterize 30 unrelated individuals of the giant freshwater prawn. The polymerase chain reaction amplification products of these eight microsatellite loci were polymorphic with the number of alleles ranging from two to 10 alleles per locus while the levels of heterozygosity ranged from 0.6333 to 0.8667 .
\end{abstract}

Keyword: 5'-anchored polymerase chain reaction; Heterozygosity; Macrobrachium rosenbergii; Microsatellite loci; Polymorphisms; RAMs 